01,05

\title{
Динамика пространственно неоднородной спиновой поляризации неравновесных электронов проводимости в магнитных переходах
}

\author{
(C) Е.A. Вилков ${ }^{1}$, Г.М. Михайлов ${ }^{2}$, С.А. Никитов ${ }^{1}$, А.P. Сафиин ${ }^{3}$, М.В. Логунов ${ }^{1}$, \\ V.N. Korenivskii ${ }^{4}$, С.Г. Чигарев ${ }^{1}$, Л.A. Фомин ${ }^{2}$ \\ ${ }^{1}$ Институт радиотехники и электроники им. В.А. Котельникова РАН, \\ Москва, Россия \\ ${ }^{2}$ Институт проблем технологии микроэлектроники и особо чистых материалов РАН, \\ Черноголовка, Россия \\ ${ }^{3}$ Национальный исследовательский университет „МЭИ“, \\ Москва, Россия \\ ${ }^{4}$ Royal Institute of Technology, \\ Stockholm, Sweden \\ E-mail: e-vilkov@yandex.ru
}

Поступила в Редакцию 11 октября 2018 г.

В окончательной редакции 19 декабря 2018 г.

Принята к публикации 17 октября 2018 г.

С учетом пространственной неоднородности распределения носителей заряда получено уравнение динамики движения магнитного момента, усредненного по ансамблю неравновесных спин-инжектированных электронов в ферромагнитном переходе, при наличии обменного взаимодействия, взаимодействия с внешним электромагнитным полем и с термостатом. Показано, что учет пространственной неоднородности распределения носителей заряда в уравнении динамики движения магнитного момента позволяет описать различные процессы переноса электронов в магнитном переходе. Оценена вероятность квантовых переходов электронов с противоположными направлениями спина, определяющих спиновую релаксацию при взаимодействии с термостатом. Показано, что анизотропия среды излучения определяется не только анизотропией тензора $s d$-обмена, но также и дополнительной анизотропией, связанной с производными этого тензора по импульсу электронов. Рассмотренные явления имеют большой потенциал для обнаружения новых эффектов и разработки на их основе новых устройств, в том числе компактных перестраиваемых источников излучения в заведомо трудном для генерации терагерцовом диапазоне частот.

Работа выполнена при частичной финансовой поддержке Российского фонда фундаментальных исследований (18-57-76001 ЭРА_а, 18-29-27020 мк), гранта Президиума РАН, тема № 0030-2017-0003, государственного задания № 007-00220-18-00.

DOI: 10.21883/FTT.2019.06.47674.279

\section{1. Введение}

В настоящее время магнитные гетероструктуры с протекающим по ним спин-поляризованным током являются одним из основных объектов исследований в спинтронике и спин-фотонике [1-11], что связано с перспективами многочисленных применений в терагерцовой спинтронике [12], в трехмерных наномагнитных устройствах [13], в запоминающих устройствах и сенсорах [14].

В таких гетероструктурах ток, протекающий в магнитном переходе, спин-поляризуется, что может приводить к значительному неравновесному накоплению спина в ограниченном объеме ферромагнитного материала. Спиновая релаксация неравновесных электронов проводимости может происходить через взаимодействие с ферромагнитной решеткой, возбуждая осцилляции намагниченности или переключая намагниченность; механизм передачи крутильного момента спина электрона магнитной решетке предложен в пионерских работах $[15,16]$.
В меньшей степени изучено взаимодействие спинов электронов проводимости и магнитной решетки ферромагнетика при резонансных условиях для релаксационных процессов (в том числе и фотоэмиссионных), вызванных переворотом спина проводящих $(s)$ электронов [17-19]. Такое взаимодействие мы и рассматриваем подробно в настоящей статье, поскольку оно требует более детального описания и объяснения в связи с перспективами применения в устройствах терагерцового диапазона частот $[18,20]$. По аналогии с предложенным в [19-22] подходом, мы рассматриваем зону проводимости ферромагнитного металла, обменно расщепленную на спиновые подзоны с некоторым энергетическим зазором. При этом зона проводимости считается неравновесной по спину из-за протекающих токов. Значительной неравновесной заселенности спиновых подзон можно достичь эффективной спиновой инжекцией, используя металлические магнитные и магнито-туннельные переходы [23-28]. При этом, в отличие от нашей работы [19], мы полагаем здесь, что возникающая при инжекции 
неравновесная спиновая поляризация электронов пространственно неоднородна, что более точно соответствует реальной экспериментальной ситуации [28] и может позволить описывать различные процессы переноса электронов в магнитном переходе.

Спин-релаксационные переходы электронов проводимости между спиновыми подзонами ферромагнетика стимулированы опосредованно через $s d$-обменное взаимодействие с электромагнитной волной. Такие спинфлип переходы электронов могут сопровождаться испусканием или поглощением фотонов $[28,29]$ с частотой, определяемой энергией эффективного обменного расщепления спиновых подзон. Для ряда переходов энергия расщепления спиновых подзон соответствует энергии фотонов терагерцового диапазона частот, что вызывает повышенный интерес с точки зрения технических применений гетероструктур как основы компактных терагерцовых источников излучения, в том числе работающих при комнатной температуре.

Для более корректного описания механизма излучения фотонов в настоящей работе предлагается модель спин-флип переходов на основе вывода кинетических уравнений, определяющих временную зависимость изменения среднего магнитного момента неравновесных инжектированных электронов с учетом их пространственной неоднородности распределения. Для моделирования спиновой релаксации инжектированных неравновесных электронов рассматривается задача динамики начального состояния электронов с учетом их спина, которое изменяется со временем под влиянием $s d$-обмена, внешнего электромагнитного поля и нерадиационных процессов релаксации. При этом состояние инжектированных электронов с разным направлением спина рассматривается как квантовая смесь. Это, в свою очередь, оказывает существенное влияние на вероятность квантовых спинфлип переходов.

\section{2. Модель магнитного перехода}

Далее, следуя работе [19], рассмотрим схему типичного магнитного перехода, представленного на рис. 1. Здесь мы рассматриваем ситуацию, когда неравновесная спиновая заселенность в ферромагнетике достигается с помощью эффективной спиновой инжекции при протекании электрического тока. При этом мы при выводе исходных уравнений учтываем зависимость неравновесной спиновой поляризации от координат $x, y, z$ [27] и считаем, что изменение начального состояния спиновой поляризации со временем происходит благодаря приложению кратковременного импульса тока инжекции. Импульс электрического тока протекает через границу однородных металлических ферромагнетиков I и II, векторы намагниченности которых $\mathbf{M}_{1}$ и $\mathbf{M}_{2}$ параллельны осям квантования в средах I и II; $\theta-$ угол между векторами $\mathbf{M}_{1}$ и $\mathbf{M}_{2}$ (рис. 1).

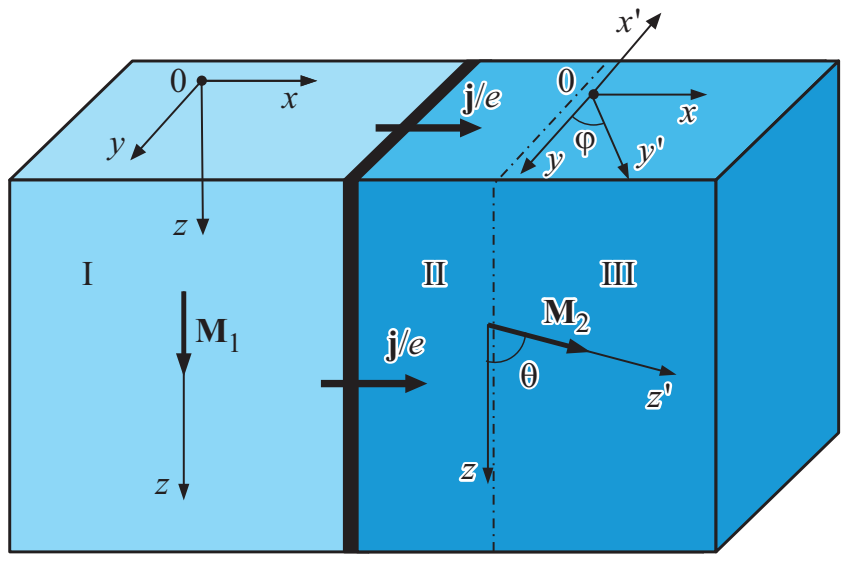

Рис. 1. Схема магнитного спин-инжекционного перехода. I - первый ферромагнетик, играющий роль инжектора; II инжекционная область второго ферромагнетика; III — часть второго ферромагнетика вне области инжекции.

Будем считать, что между слоями ферромагнетиков нанесен сверхтонкий буферный слой (диэлектрик или немагнитный металл), устраняющий обменное взаимодействие между ними. При прохождении тока через спин-инжекционный переход из первого ферромагнетика (область I) происходит инжекция нескомпенсированных спинов в область II второго ферромагнетика, затем инжектированные носители заряда стекают через область III, выполняющую роль коллектора. Изменение квазиуровней Ферми для средних заселенностей спиновых подзон в областях I, II и II (с обозначением энергетических релаксационных переходов электронов с переворотом спина в области II) показано на рис. 2 [30]. В терагерцовом диапазоне частот можно приближенно считать, что колебания намагниченности магнитной решетки ферромагнетиков слабо выражены, поскольку типичные собственные частоты таких колебаний лежат в пределах $\sim 10^{10}-10^{11} \mathrm{~s}^{-1}$. Поэтому в первом приближении допустимо считать магнитную решетку неподвижной. Кроме того, колебания и перемагничивание решетки наблюдаются в слоях магнетиков порядка нескольких нанометров. В настоящей работе мы предполагаем, что слои рабочей области много больше этого размера, поэтому энергетически выгодным становится состояние со стационарным направлением намагниченности ферромагнетика. Таким образом, считается, что процессы передачи крутильного момента от электронов проводимости магнитной решетке и влияние встроенных полей из-за неравновесной аккумуляции спинов либо пренебрежимо малы из-за малой плотности тока инжекции и неравновесной аккумуляции спинов, либо такие процессы (например, перемагничивание магнитной решетки) уже произошли. В этом случае магнитная решетка характеризуется стационарной намагниченностью, при этом частоты спиновой подсистемы электронов проводимости много больше, чем частоты 


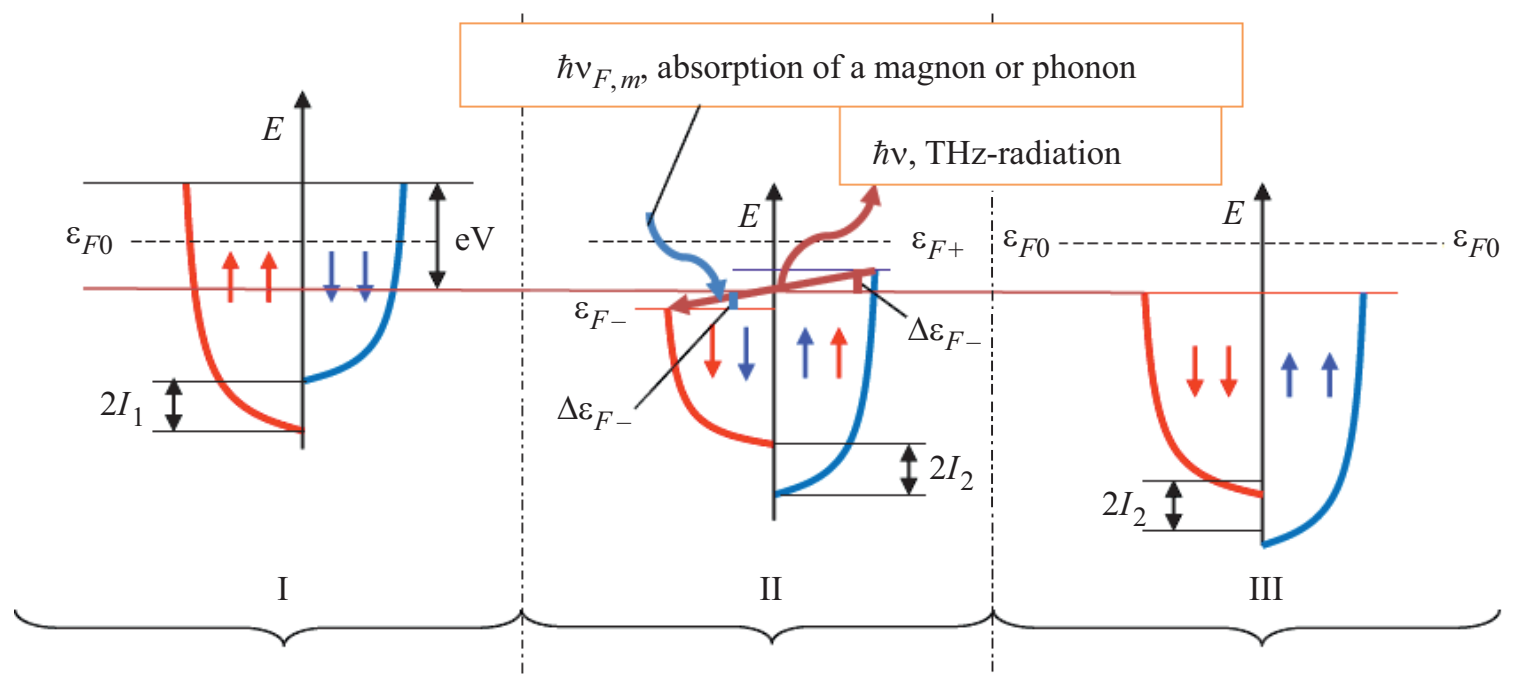

Рис. 2. Схема энергетических электронных зон и квазиуровней Ферми: I - в первом ферромагнетике, II - для неравновесных электронов во втором ферромагнетике, III - для равновесных электронов во втором ферромагнетике вне области инжекции.

для $3 d$-электронов, что позволяет пренебречь динамикой магнитной решетки на таких частотах.

В используемой нами модели принимаем, что электроны проводимости имеют изотропный параболический спектр энергий с некоторой эффективной массой. Учитывается воздействие на них обменного взаимодействия с $d$-электронами ( $s d$-обмен), внешнего электромагнитного поля и внешней равновесной системы „термостата“. Обменное взаимодействие $s$-электронов, определяющих проводимость ферромагнитного металла, с $d$-электронами, „локализованными“ на атомах кристаллической решетки, зависит от спинового состояния $s$ - и $d$-электронов, при этом спиновое состояние $d$-электронов считается связанным с намагниченностью кристаллической решетки ферромагнетика. Такое взаимодействие описывается по механизму $s d$-обмена [31]. Вследствие этого взаимодействия обменная энергия $s$-электронов зависит от направления их спинов относительно намагниченности кристаллической решетки ферромагнетика. Учет этого достигается введением спиновой переменной в гамильтониан взаимодействия. При токовой инжекции спин-поляризованных электронов в условиях достаточно большой длины релаксации спинов $l$ их продольную релаксацию импульса можно считать быстрой, в отличие от спиновой релаксации, когда спин-инжектированные электроны сохраняют какое-то время спиновое состояние, приобретенное в инжекторе (в нашем случае - в первом ферромагнетике, см. область II на рис. 2). Область сохранения спинового состояния выделена как область II на рис. 2. В этой области с иной, чем в инжекторе, намагниченностью $M_{2}$ инжектированные электроны с противоположными спинами образуют две квазиравновесные группы. При рассмотрении средних заселенностей инжектированных электронов по спиновым подзонам, можно ввести для них квазистаци- онарные уровни Ферми в каждой из подзон как добавку к равновесному значению уровня Ферми. Кроме этого, нужно учесть условие электронейтральности так, чтобы общее число электронов в рабочей области оставалось постоянным. В результате возникает разница в положениях квазиуровней в спиновых подзонах и, таким образом, при инжекции спинов током в магнитном переходе может возникнуть отрицательная спиновая температура, предсказанная в [32].

\section{3. Гамильтониан инжектированных электронов}

Введем систему координат $x^{\prime}, y^{\prime}, z^{\prime}$, в которой ось $z^{\prime}$ направленна вдоль вектора намагниченности $\mathbf{M}_{2}$ (рис. 1). На рис. $1 \theta, \varphi-$ полярный и азимутальный углы оси $z^{\prime}$ по отношению к осям $x, y, z$ в инжекторе. Переход от одной системы координат к другой соответствует повороту на угол $\varphi$ вокруг оси $z$ и затем - на угол $\theta$ вокруг нового положения оси $y^{\prime}$. В системе отсчета $x^{\prime}, y^{\prime}, z^{\prime}$ для квантовой смеси инжектированных электронов с разными направлениями спинов [33,34] матрица плотности описывается в виде [19]

$$
\rho=\left(\begin{array}{cc}
\frac{1}{2}+\frac{P}{2} \cos \theta & -\frac{P}{2} \sin \theta \\
-\frac{P}{2} \sin \theta & \frac{1}{2}-\frac{P}{2} \cos \theta
\end{array}\right) .
$$

Здесь $P-$ степень спиновой поляризации инжектированных электронов в начальный момент времени. Для инжектированных электронов матрица плотности является недиагональной. Таким образом, мы должны описывать состояния инжектированных электронов с разными направлениями спинов как квантовую смесь. Наличие поперечных спиновых компонент может оказать существенное влияние на вероятность квантовых спин-флип переходов, что и будет показано далее. 
Будем следовать приближению, используемому в работе [23]. Гамильтониан одного инжектированного электрона с учетом взаимодействия с электромагнитной волной для уравнения Паули можно представить в виде

$$
\hat{H}=\hat{H}_{k}+\hat{H}_{s d}+\hat{H}_{A},
$$

где $\hat{H}_{k}-$ оператор кинетической энергии электрона, равный $\hat{p}^{2} / 2 m^{*}, \hat{p}$ - оператор импульса, умноженный на единичную матрицу $2 \times 2$. Это дает параболическую дисперсию зависимости энергии от импульса электрона с некоторой эффективной массой электрона $m^{*} . \hat{H}_{s d}-$ оператор $s d$-обменного взаимодействия. В общем виде он задается выражением

$$
\hat{H}_{s d}=-\hat{\boldsymbol{\sigma}} \mu_{B} \mathbf{H}_{s d}^{0}
$$

где векторная матрица, расположенная по ортам осей координат равна $\hat{\boldsymbol{\sigma}}=\mathbf{x} \hat{\sigma}_{x}+\mathbf{y} \hat{\sigma}_{y}+\mathbf{z} \hat{\sigma}_{z}, \mathbf{H}_{s d}^{0}-$ встроенное эффективное $s d$-обменное поле, а $\mu_{B}-$ магнетон Бора. $\hat{H}_{A}$ - оператор взаимодействия с электромагнитной волной с векторным потенциалом А. Он определяется [23], как

$$
\hat{H}_{A}=-\hat{\boldsymbol{\sigma}} \mu_{B} \mathbf{H}_{A},
$$

где индуцированное электромагнитной волной $s d$-обменное поле равно

$$
\mathbf{H}_{A}=\frac{e}{2 c}\left(\frac{\partial \mathbf{H}_{s d}^{0}}{\partial \hat{\mathbf{p}}} \mathbf{A}+\mathbf{A} \frac{\partial \mathbf{H}_{s d}^{0}}{\partial \hat{\mathbf{p}}}\right) .
$$

Используя первые два члена гамильтониана (2), получаем решение в виде спиноров со спином „верх“ или „вниз““, соответствующих двум параболическим спиновым подзонам, расщепленных по энергии на величину $2 \mu_{B}\left|\mathbf{H}_{s d}^{0}\right|$. Третий член гамильтониана (2) можно рассматривать как возмущение, вызывающее излучательные переходы между спиновыми подзонами с переворотом спина электрона под действием электромагнитной волны с вектор-потенциалом А. Координатная часть спиноров начального и конечного состояния с учетом импульса и энергии фотона дает сохранение суммарного импульса, а временная часть - сохранение энергии. Спин излученного фотона равен единице (или -1$)$, что соответствует изменению спина электрона в результате излучательного перехода с переворотом его спина.

Из-за того, что редуцированное состояние инжектированного электрона соответствует спинору со спином „вверх“ (или „вниз“") из структуры формул (3) и (4) следует очевидное следствие. Если встроенное и индуцированное $s d$-обменные эффективные поля коллинеарны, то переходы с переворотом спина невозможны с участием таких состояний электрона. Действительно, выбирая ось $z$ квантования вдоль вектора $\mathbf{H}_{s d}^{0}$, получаем, что для вектора $\mathbf{H}_{A}$ отлична от нуля только его $z$-компонента (из-за коллинеарности с вектором $\mathbf{H}_{s d}^{0}$ ), а в возмущении (4) будет присутствовать только $z$-матрица Паули. Однако с ее помощью невозможно построить возмущение, приводящее к изменению проекции спина электрона на ось $z$ в редуцированных состояниях $(1,0)$ и $(0,1)$, поскольку она диагональна в выбранной системе координат.

Рассмотрим более подробно образование встроенного и индуцированного эффективных $s d$-полей. Поскольку для переходных металлов спин атома определяется главным образом спином $d$-электронов, то используем формулу для оценки обменного $s d$-взаимодействия [31]:

$$
\mathbf{H}_{s d}^{0}=\alpha_{s d} \mathbf{M}_{2}
$$

где $\alpha_{s d}-$ константа $s d$-обменного взаимодействия, а $\mathbf{M}_{2}$ - намагниченность среды инжекции. Формула (6) связывает два вектора $\mathbf{H}_{s d}^{0}$ и $\mathbf{M}_{2}$ через умножение на константу $\alpha_{s}$, что характерно для изотропной (кубической) среды. Обобщая эту формулу и вводя тензор $s d$-обмена, получаем

$$
\left(\mathbf{H}_{s d}^{0}\right)_{k}=\left(\alpha_{s d}\right)_{k l}\left(\mathbf{M}_{2}\right)_{l},
$$

где по одинаковым индексам идет суммирование. Легко показать, используя формулу (5) для вычисления индуцированного электромагнитной волной $s d$-обменного поля $\mathbf{H}_{A}$ с помощью формулы (7) или (6), что векторы $\mathbf{H}_{s d}^{0}$ и $\mathbf{H}_{A}$ коллинеарные, если среда изотропная. В то же время различие между собой диагональных компонент тензора $s d$-обмена (при приведении к главным осям тензора) приводит к неколлинеарности встроенного и индуцированного эффективных полей, что и определяет анизотропную среду излучения. Лишь в этом случае возможны излучательные переходы с переворотом спина электронов, находящихся в редуцированных состояниях, в рамках рассматриваемой модели. Это существенно меняет рассмотрение процесса спин-инжекционного излучения по сравнению с [23].

Согласно формулам (3)-(5) можно ввести для удобства дальнейших преобразований вектор $\mathbf{I}$, имеющий размерность энергии, через встроенное эффективное $s d$-обменное поле $\mathbf{H}_{s d}^{0}$ :

$$
\mathbf{I}=\hbar \gamma \frac{\mathbf{H}_{s d}^{0}}{2}=\mu_{B} G(\mathbf{p}) \mathbf{M}_{2}(\mathbf{r})
$$

где $G(\mathbf{p})=\left(\alpha_{s d}\right)_{i j}(\mathbf{p})-$ тензор $s d$-обмена (отметим, что в тонких пленках из материалов даже с кубической симметрией в объемных кристаллах могут возникать выделенные направления анизотропии [31]), r - радиусвектор, $\gamma$ - гиромагнитное отношение. Так же можно ввести вектор $\mathbf{J}$ через индуцированное электромагнитной волной $s d$-обменное поле $\mathbf{H}_{A}$

$$
\mathbf{J}=\hbar \gamma \frac{\mathbf{H}_{e}}{2}=\frac{e}{2 c}\left(\frac{\partial \mathbf{I}}{\partial \hat{\mathbf{p}}} \mathbf{A}+\mathbf{A} \frac{\partial \mathbf{I}}{\partial \hat{\mathbf{p}}}\right) .
$$

Через эти векторы выразим новый вектор $\mathbf{H}_{s d}$, определяющий $s d$-обменное поле с учетом добавки от внешнего электромагнитного поля. Этот вектор имеет вид

$$
\mathbf{H}_{s d}=2(\mathbf{I}-\mathbf{J}) / \hbar \gamma
$$


Отметим, что, помимо координатной составляющей, поле $\mathbf{H}_{s d}$ включает временную составляющую $\mathbf{J}$ с частотой колебания, задаваемой внешним электромагнитным полем. Частоту внешнего поля мы полагаем равной частоте перехода между подзонами $\omega_{1,2} \approx\left(2 I_{z}\right) / \hbar$. Если частота внешнего поля сравнима с частотой перехода между подзонами (которая на несколько порядков превосходит частоту ферромагнитного резонанса пленки), то намагниченность решетки, как мы уже отмечали, не успевает следовать за внешним полем и сохраняет свое исходное положение. Далее мы пренебрегаем динамикой магнитной решетки. Ограничимся рассмотрением только поперечных компонент $\mathbf{A}(z, t)$, например, компонент $A_{x}(z, t)$ и $A_{y}(z, t)$. Таким образом, гамильтониан неравновесных инжектированных электронов будет определяться обменным полем, которое модулировано внешним излучением. Анизотропия обменного взаимодействия и наличие внешнего поля приводят к существованию в гамильтониане (2) слагаемого, которое описывает возмущение и содержит недиагональные компоненты, приводящие к перевороту спина.

\section{4. Аналог уравнения Блоха для инжектированных электронов с учетом транспорта электронов}

Для описания кинетики изменения намагниченности инжектированных электронов удобно вывести аналог модифицированного (учитывается транспортный член) уравнения Блоха с учетом взаимодействия с электромагнитным полем для инжектированных электронов, что позволили исследовать с его помощью не только релаксационные, но также и излучательные процессы, а также транспорт неравновесных спинов. Поскольку уравнение Паули при взаимодействии с внешним магнитным полем аналогично по структуре уравнению (2), то вывод аналога уравнения Блоха такой же, как приводится, например, в монографии [33]. Вывод основан на использовании матрицы плотности для определения среднего вектора поляризации электрона при однородной плотности спинов, который в дальнейшем трансформируется в намагниченность инжектированных электронов. Однако для учета транспортного члена в аналоге уравнения Блоха необходимо принять во внимание пространственную неоднородность спинового распределения.

Для этого, в отличие от вектор-оператора спиновой поляризации, введем для рассмотрения оператор плотности спинового распределения. Его $n$-ную компоненту $\boldsymbol{\pi}_{n}$ представим через произведение оператора компоненты спиновой поляризации и оператора плотности частицы в эрмитовосопряженной форме

$$
\pi_{n}=\frac{1}{2}\left(\sigma_{n} \delta\left(\mathbf{r}-\mathbf{r}_{o}\right)+\delta\left(\mathbf{r}-\mathbf{r}_{o}\right) \sigma_{n}\right)
$$

где номера матриц Паули $n=1-3$ соответствуют компонентам $x, y, z$ и введена дельта-функция по координатам, $\mathbf{r}_{o}$ - радиус-вектор частицы. В формуле (11) и далее над операторами мы не пишем значок оператора. Среднее значение этого оператора по всему ансамблю равно

$$
\left\langle\pi_{n}\right\rangle=\operatorname{Sp}\left(\pi_{n}, \rho\right),
$$

где $\rho-$ матрица плотности для инжектированных электронов. Производная этого среднего значения определяется как

$$
\frac{d\left\langle\pi_{n}\right\rangle}{d t}=\operatorname{Sp}\left(\pi_{n} \frac{d \rho}{d t}\right)=\frac{1}{i \hbar} \operatorname{Sp}\left(\pi_{n}[h, \rho]\right)=\frac{1}{i \hbar} \operatorname{Sp}\left(\left[\pi_{n}, H\right] \rho\right),
$$

где в (13) скобками [, ] обозначили коммутатор с гамильтонианом $(2)-(5)$, который запишем в виде

$$
\hat{H}=\frac{\mathbf{p}^{2}}{2 m^{*}}-\mu_{B}\left(\mathbf{H}_{s d}^{0}+\mathbf{H}_{A}\right) \boldsymbol{\sigma} .
$$

В уравнении (13) использовали уравнение для матрицы плотности и возможность циклической перестановки операторов при вычислении следа произведения. Так как

$$
\left[\sigma_{n}, \delta\left(\mathbf{r}-\mathbf{r}_{o}\right)\right]=0 \text { и }\left[\sigma_{n}, \frac{\mathbf{p}^{2}}{2 m^{*}}\right]=0,
$$

то коммутатор в последнем равенстве (13) упрощается и разбивается на две части

$$
\begin{aligned}
{\left[\pi_{n}, \hat{H}\right]=} & \sigma_{n}\left[\delta\left(\mathbf{r}-\mathbf{r}_{o}\right), \frac{\mathbf{p}^{2}}{2 m^{*}}\right] \\
& -\delta\left(\mathbf{r}-\mathbf{r}_{o}\right)\left[\sigma_{n}, \mu_{B}\left(\mathbf{H}_{s d}^{0}+\mathbf{H}_{A}\right) \boldsymbol{\sigma}\right] .
\end{aligned}
$$

Рассмотрим их по отдельности, используя известное правило умножения матриц Паули

$$
\sigma_{n} \sigma_{m}=i \varepsilon_{n m k} \sigma_{k}+\delta_{n m} \sigma_{o},
$$

где по одинаковым индексам проводится суммирование (здесь и далее), $\varepsilon_{n m k}$ - антисимметричный единичный тензор (символ Леви-Чивиты), $\delta_{n m}-$ симметричный единичный тензор (символ Кронекера), а $\sigma_{o}$ - единичная матрица $2 \times 2$. Для первой части (15) получаем

$$
\begin{aligned}
\sigma_{n}\left[\delta\left(\mathbf{r}-\mathbf{r}_{0}\right), \frac{\mathbf{p}^{2}}{2 m^{*}}\right] & =\sigma_{n}\left[\frac{1}{2 m^{*}}\left\{\delta\left(\mathbf{r}-\mathbf{r}_{0}\right), p_{k}\right\}, p_{k}\right] \\
& \left.=\sigma_{n} \Pi_{k}, p_{k}\right],
\end{aligned}
$$

где фигурные скобки $\{$,$\} обозначают антикоммутатор,$ при этом оператор

$$
\Pi_{k}=\frac{1}{2 m^{*}}\left\{\delta\left(\mathbf{r}-\mathbf{r}_{0}\right), p_{k}\right\},
$$

как легко видеть, есть оператор плотности потока частиц. Для второй части следует, что

$$
\begin{aligned}
-\delta\left(\mathbf{r}-\mathbf{r}_{0}\right) & {\left[\sigma_{n}, \mu_{B}\left(\mathbf{H}_{s d}^{0}+\mathbf{H}_{A}\right) \boldsymbol{\sigma}\right] } \\
& =-\delta\left(\mathbf{r}-\mathbf{r}_{0}\right) \mu_{B}\left(\mathbf{H}_{s d}^{0}+\mathbf{H}_{A}\right){ }_{j} 2 i \varepsilon_{n j k} \sigma_{k} .
\end{aligned}
$$


Подставляя в последнее равенство (13) части (17) и (19), получаем

$$
\begin{aligned}
& \frac{1}{i \hbar} \operatorname{Sp}\left(\left[\pi_{n}, H\right] \rho\right)=\frac{1}{i \hbar} \operatorname{Sp}\left(\left(\sigma_{n}\left[\Pi_{k}, p_{k}\right]\right.\right. \\
& \left.\left.-\delta\left(\mathbf{r}-\mathbf{r}_{0}\right) \mu_{B}\left(\mathbf{H}_{s d}^{0}+\mathbf{H}_{A}\right)_{j} 2 i \varepsilon_{n j k} \sigma_{k}\right) \rho\right) \\
& =\frac{1}{i \hbar} \operatorname{Sp}\left(\sigma_{n}\left[\Pi_{k}, p_{k}\right] \rho\right)-\frac{1}{i \hbar} \operatorname{Sp}\left(\left(\mu_{B}\left(\mathbf{H}_{s d}^{0}+\mathbf{H}_{A}\right)_{j} 2 i \varepsilon_{n j k} \pi_{k}\right) \rho\right) .
\end{aligned}
$$

Первый член в последнем равенстве (20) можно (как легко показать прямым преобразованием, используя эрмитовость оператора $\mathbf{p}^{2}$ ) переписать в виде

$$
\frac{1}{i \hbar} \operatorname{Sp}\left(\left[\Pi_{k}, p_{k}\right] \sigma_{n} \rho\right)=-\nabla_{k} \operatorname{Sp}\left(\Pi_{k} \sigma_{k} \rho\right)=-\nabla_{k}\left\langle\Pi_{k} \sigma_{n}\right\rangle,
$$

т. е., как дивергенцию тензора средней плотности потока поляризации, а второй член в последнем равенстве (20), считая длину волны электромагнитного поля, определяющую пространственный масштаб изменения $\left(\mathbf{H}_{A}\right)_{j}$, много большей длины спиновой релаксации, что заведомо выполняется для терагерцового диапазона длин волн, как

$$
\begin{aligned}
-\frac{1}{i \hbar} \operatorname{Sp}\left(\left(\mu _ { B } \left(\mathbf{H}_{s d}^{0}\right.\right.\right. & \left.\left.\left.+\mathbf{H}_{A}\right)_{j} 2 i \varepsilon_{n j k} p i_{k}\right) \rho\right) \\
& \approx-\frac{2 \varepsilon_{n j k}}{\hbar} \mu_{B}\left(\mathbf{H}_{s d}^{0}+\mathbf{H}_{A}\right)_{j} \operatorname{Sp}\left(\pi_{k} \rho\right) \\
& =\frac{2}{\hbar}\left(\langle\boldsymbol{\pi}\rangle \times \mu_{B}\left(\mathbf{H}_{s d}^{0}+\mathbf{H}_{A}\right)\right)_{n} .
\end{aligned}
$$

Подставляя результаты из (21) и (22) в (13), получаем уравнение для средней плотности поляризации

$$
\frac{d\langle\boldsymbol{\pi}\rangle_{n}}{d t}=-\nabla_{k}\left\langle\Pi_{k} \sigma_{n}\right\rangle+\frac{2}{\hbar}\left(\langle\boldsymbol{\pi}\rangle \times \mu_{B}\left(\mathbf{H}_{s d}^{0}+\mathbf{H}_{A}\right)\right) .
$$

Используем далее для компактности записи суммарное (встроенное и наведенное) эффективное обменное поле. Более того, добавим к этому очевидную „обычную“ магнитную напряженность $\mathbf{H}_{H}$ электромагнитной волны, действующую на спин электрона, аналогично используемой в ЭПР спектроскопии. Подстановка напряженности вместо индукции магнитного поля объясняется использованием больших частот электромагнитной волны относительно собственных частот магнитной решетки ферромагнетика, что приводит к магнитной проницаемости, равной единице. В результате:

$$
\mathbf{H}_{s d}=2 \mu_{B}\left(\mathbf{H}_{s d}^{0}+\mathbf{H}_{A}+\mathbf{H}_{H}\right) / \hbar \gamma
$$

Добавка компоненты напряженности магнитного поля электромагнитной волны $\mathbf{H}_{H}$ в (24) связано с тем, что в изотропной среде действие $\mathbf{H}_{A}$ не приводит к излучательным процессам с переворотом спина, как это обсуждалось ранее, и такие излучательные процессы определяются в этом случае именно компонентом $\mathbf{H}_{H}$.
Далее из пропорциональности плотности поляризации и намагниченности, а также вводя феноменологически поперечное и продольное затухание из-за безизлучательных спиновых релаксаций при взаимодействии со средой, получаем аналог уравнения Блоха с транспортной частью для намагниченности инжектированных электронов, в котором учитывается транспорт и взаимодействие электронов проводимости с электромагнитной волной через $s d$-взаимодействие:

$$
\begin{array}{r}
\frac{d \boldsymbol{\mu}_{\perp}}{d t}=\gamma\left(\boldsymbol{\mu} \times \mathbf{H}_{s d}\right)_{\perp}-\nabla_{k}\left\langle\Pi_{k} \mu_{\perp}\right\rangle-\frac{\boldsymbol{\mu}_{\perp}}{\tau_{\perp}}, \\
\frac{d \boldsymbol{\mu}_{\|}}{d t}=\gamma\left(\boldsymbol{\mu} \times \mathbf{H}_{s d}\right)_{\|}-\nabla_{k}\left\langle\Pi_{k} \mu_{\|}\right\rangle-\frac{\boldsymbol{\mu}_{\|}-\left\langle\boldsymbol{\mu}_{\|}\right\rangle}{\tau_{\|}},
\end{array}
$$

где $\tau_{\|}$- время продольной спиновой релаксации инжектированных электронов, а $\tau_{\perp}-$ время релаксации поперечной составляющей спина, которую мы приписываем действию механизма Слончевского-Берже [16]. Время релаксации поперечной составляющей спина мало, и релаксация происходит при распространении волны на расстояние порядка единиц нанометров [16]. Время продольной спиновой релаксации намного больше, и релаксация происходит при распространении волны на расстояние в несколько десятков нанометров. Из системы уравнений (25) видно, что состояние инжектированных неравновесных электронов будет изменяться со временем под влиянием $s d$-обмена, внешнего электромагнитного поля, нерадиационных процессов релаксации и процессов переноса носителей заряда, а также транспорта электронов.

Можно легко показать, что дивергенция от спинового потока $\nabla_{k}\left\langle\Pi_{k} \mu_{\perp}\right\rangle, \nabla_{k}\left\langle\Pi_{k} \mu_{\|}\right\rangle$определяется через парциальные плотности тока электронов с разными направлениями спина [6]:

$$
\nabla_{k}\left\langle\Pi_{k} \mu_{\perp}\right\rangle, \quad \nabla_{k}\left\langle\Pi_{k} \mu_{\|}\right\rangle \sim \frac{\hbar}{2 e}\left[j_{+}(x)-j_{-}(x)\right] .
$$

Кроме того, зная, как эти токи зависят от электрического поля $E(x)$ и градиентов парциальных концентраций в квазиклассическом приближении [6]

$$
j_{ \pm}=e \mu_{ \pm} n_{ \pm}(x) E(x)-e D_{ \pm} \frac{d n_{ \pm}}{d x},
$$

где $\mu_{ \pm}$и $D_{ \pm}$- парциальные подвижности и коэффициенты диффузии электронов, приходим к выводу, что, в отличие от нашей предыдущей работы [19], полученные в этой работе уравнения динамики магнитного момента (25) одновременно описывают и различные процессы переноса (диффузия, дрейф и др.) электронов в магнитном переходе (см., например работу [30]).

Проведем оценку динамики магнитного момента неравновесного электрона для объектов с размерами меньше длины спиновой релаксации, что позволяет пренебречь транспортными слагаемыми $\nabla_{k}\left\langle\Pi_{k} \mu_{\perp}\right\rangle$, $\nabla_{k}\left\langle\Pi_{k} \mu_{\|}\right\rangle$в уравнениях (25). Это означает, что мы 
пренебрегаем зависимостью неравновесной спиновой поляризации от координат $x, y, z$ [30]. Кроме этого, пренебрежем также вкладом $\mathbf{H}_{H}$ в $(24)$, считая среду оптимально анизотропной с превалирующим вкладом $\mathbf{H}_{A}$.

Рассмотрение стационарного уравнения для продольной компоненты магнитного момента (спиновая поляризация) с учетом ее пространственной неоднородности проводился в работе [30]. Далее введем компоненты магнитного момента в соответствии с рис. 1 следующим образом:

$$
\mu_{z}=\left\langle\mu_{\|}\right\rangle, \quad \mu_{x, y}=\left\langle\mu_{\perp}\right\rangle_{x, y} .
$$

Заметим, что магнитный момент инжектированных электронов $\mu_{z}$, в сравнении его с равновесным значением вдоль направления намагниченности пленки, является мерой неравновесной средней заселенности в спиновых подзонах при данном значении импульса инжектированного электрона. Что касается проекций магнитного момента $\mu_{x}$ и $\mu_{y}$, то они определяются недиагональными элементами матрицы плотности, которые описывают переходы между спиновыми подзонами.

Приняв во внимание соотношения (26) и равенство $\omega_{12}-\gamma H_{A}^{(z)}=2 I_{z} / \hbar-\gamma H_{A}^{(z)}=\gamma H_{s d}^{(z)}$, перейдем от системы (25) к системе уравнений для составляющих вектора $\boldsymbol{\mu}$ :

$$
\begin{gathered}
\frac{d \mu_{x}}{d t}+\frac{\mu_{x}}{\tau_{\perp}}=-\gamma \mu_{z} H_{A}^{(y)}+\left(\omega_{12}-\gamma H_{A}^{(z)}\right) \mu_{y} ; \\
\frac{d \mu_{y}}{d t}+\frac{\mu_{y}}{\tau_{\perp}}=\gamma H_{A}^{(x)} \mu_{z}-\left(\omega_{12}-\gamma H_{A}^{(z)}\right) \mu_{x} ; \\
\left.\frac{d \mu_{z}}{d t}+\frac{\mu_{z}-\mu_{z}^{2}}{\tau_{\|}}=\gamma\left[-H_{A}^{(x)} \mu_{y}+H_{A}^{(y)}\right) \mu_{x}\right],
\end{gathered}
$$

где $\mu_{z}^{e}-$ продольный магнитный момент электрона в состоянии равновесия, $H_{A}^{(z)}=(\gamma \hbar / 2) J_{z}-z$-компонента поля $\mathbf{H}_{A}$ (9). В формулах (27) $\omega_{12}-$ резонансная частота перехода между уровнями $\varepsilon_{F+}$ и $\varepsilon_{F-}$ (рис. 2), $\omega_{12}=\left(\frac{p_{1}^{2}}{2 m}-\frac{p_{2}^{2}}{2 m}+2 I_{z}\right)$. В (27) учтено, что при выборе оси квантования вдоль вектора I поперечные компоненты эффективного обменного поля $\mathbf{H}_{s d}(24)$ полностью определяются поперечными компонентами внешнего поля $\mathbf{H}_{A}$. Поскольку мы рассматриваем лишь электронную спиновую подсистему, то в нашей модели возможны только прямозонные переходы без участия третьей частицы (рис. 2), что следует из учета координатной части в матрице плотности. Поэтому частота перехода будет равна $\omega_{12} \approx\left(2 I_{z}\right) / \hbar$.

Уравнения (27) представляют собой аналог уравнений Блоха. Они описывают динамику усредненного магнитного момента инжектированных электронов в обменном поле, модулированном внешним электромагнитным полем (см. уравнения (5), (6), (10)). Несмотря на сложный характер энергетического спектра реальных веществ, для описания взаимодействия с ними монохроматического электромагнитного излучения часто оказывается возможным использование двухуровневой модели. В рамках нашей модели полученные уравнения (27) фактически являются точными (без учета релаксационных членов), поскольку гамильтониан (2), соответствующий уравнению Паули, линеен по спину инжектированного электрона. Поэтому для описания динамики магнитного момента инжектированных электронов в рамках принятой модели уравнения (27) применимы в общем случае (без релаксационных членов). Аналогичного типа уравнения применялись для экспериментального определения величин спиновых ядерных моментов парамагнетиков во внешнем магнитном поле [31,35]. Отличие заключается в том, что $s d$-обменное поле является внутренним полем ферромагнетика. При достаточном отклонении от равновесного состояния электронной спиновой поляризации возникает прецессия спинов электронов проводимости в обменном поле, описываемая уравнениями (27). Учет релаксационных членов в этих уравнениях проведен феноменологически. В общем виде такой учет релаксации имеет определенные ограничения, но часто используется, поскольку на полуколичественном уровне дает правильные результаты.

В отсутствии затухания из (25) или (27) получаем следующее уравнение движения магнитного момента

$$
\frac{d \boldsymbol{\mu}}{d t}=\gamma\left[\boldsymbol{\mu} \mathbf{H}_{s d}\right]
$$

Система уравнений (27) и ее решение описывают процесс, когда время продольной релаксации определяется интенсивностью процессов обмена энергии между инжектированными электронами и решеткой [31], а время поперечной релаксации определяется тем, как быстро нарушается синхронность прецессии магнитных диполей [15]. Релаксация поперечной компоненты спина происходит достаточно быстро в масштабе времени продольной релаксации. Именно при релаксации поперечной компоненты спина происходит передача крутильного момента спина магнитной решетке ферромагнетика. При этом могут происходить осцилляции намагниченности или переключение намагниченности ферромагнетика [1-10], которые мы в настоящей работе не учитываем. Дальнейший процесс релаксации спина связан с переходом электронов между неравновесными квазиуровнями Ферми с переворотом спина, который может приводить к терагерцовому и дальнему инфракрасному излучению. В следующем разделе оценим вероятность процесса релаксации продольной компоненты с переворотом спина.

\section{5. Скорость квантовых переходов}

Из уравнений (27) видно, что при данных начальных условиях $\left(n_{+}>n_{-}-\right.$неравновесная заселенность спиновых подзон) заселенность верхней подзоны $(+)$ убывает со временем, а заселенность нижней подзоны $(-)$ растет. Нас интересует релаксационный процесс с излучательными и безизлучательными переходами вблизи начального момента времени, когда концентрация 
инжектированных носителей максимальна (на практике вблизи границы она будет постоянной величиной за счет постоянного во времени тока инжекции). Следовательно, скорость квантовых переходов в начальный момент времени будет определять вероятность переходов (количество квантовых переходов в единицу времени), которую мы ищем. Для того, чтобы оценить скорость квантовых переходов, найдем зависимость $\mu_{z}(t)$, исходя из ряда приближений.

Поскольку $J_{z} \sim \exp \left(-i \omega_{12} t\right)$, то $z$-компонента поля $H_{s d}^{(z)}$, согласно формуле (24), зависит от времени. Тем не менее, принимая во внимание неравенство $I_{z} \gg J_{z}$, пренебрежем зависимостью от времени $z$-компоненты поля при дальнейшем анализе уравнений (27). Зависимость от времени поперечных компонент поля $H_{s d}^{(y)}, H_{s d}^{(x)}$ сохраняем, так как $J_{x} \sim \exp \left(-i \omega_{12} t\right)$ и $J_{y} \sim \exp \left(-i \omega_{12} t\right)$, то есть поперечные компоненты обменного поля полностью определяются зависящими от времени компонентами внешнего поля $\mathbf{H}_{A}$. При $\tau_{\perp} \ll \tau_{\|}$, и $H_{A}^{2(\perp)} \approx$ const, $\omega_{12} \ll 1 / \tau_{\perp}$ (при круговой поляризации внешнего электромагнитного поля выполняется точное равенство $H_{A}^{2(\perp)}=$ const) из системы уравнений (27) мы можем получить

$$
\begin{aligned}
& \mu_{z}(t) \approx\left(\mu_{z}(0)-\frac{\mu_{z}^{e}}{1+\gamma^{2} N_{A}^{2(\perp)} \tau_{\perp} \tau_{\|}}\right) \\
& \times \exp \left(-\left\{1+\gamma^{2} H_{A}^{2(\perp)} \tau_{\perp} \tau_{\|}\right\} t / \tau_{\|}\right)+\frac{\mu_{z}^{e}}{1+\gamma^{2} N_{A}^{2(\perp)} \tau_{\perp} \tau_{\|}} .
\end{aligned}
$$

Учитывая, что

$$
\mu_{z}(t)=\frac{\gamma \hbar}{2} P_{z}(t)
$$

где $P_{z}=\left(n_{+}-n_{-}\right) / n_{e l}-$ степень спиновой поляризации неравновесных электронов, и то, что скорость квантовых переходов мы оцениваем, как

$$
W=\left.n_{e l}\left(d P_{z}(t) / d t\right)\right|_{t=0},
$$

из (29) мы получим

$$
\begin{aligned}
W \approx n_{e l} \mid\left(P_{z}(0)-\frac{P_{z}^{e}}{\left(1+\gamma^{2} H_{A}^{2(\perp)} \tau_{\perp} \tau_{\|}\right)}\right) \\
\quad \times\left(\frac{1+\gamma^{2} H_{A}^{2(\perp)} \tau_{\perp} \tau_{\|}}{\tau_{\|}}\right) \mid .
\end{aligned}
$$

Здесь $n_{e l}$ - концентрация инжектированных неравновесных по спину электронов в металле, $P_{z}^{e}-$ равновесное значение спиновой поляризации.

Из формулы (30) видно, что вероятность переходов в значительной степени зависит от величины $\gamma^{2} H_{A}^{2(\perp)} \tau_{\perp} \tau_{\|}$по сравнению с единицей, т. е. неравенства $\gamma^{2} H_{A}^{2(\perp)} \tau_{\perp} \tau_{\|} \ll 1$ и $\gamma^{2} H_{A}^{2(\perp)} \tau_{\perp} \tau_{\|} \gg 1$ соответствуют двум разным режимам спиновой релаксации. Проясним физический смысл этих двух режимов следующим образом.
Из уравнения (29) следует, что частота прецессии магнитного момента электрона вокруг оси $z \omega_{p r} \approx \gamma H_{A}^{(\perp)}$. Если ввести эффективное время спиновой релаксации как $\tau_{e f f}=\sqrt{\tau_{\perp} \tau_{\|}}$, то в случае $\omega_{p r} \tau_{e f f} \ll 1$ реализуется режим низкой добротности прецессии (магнитный момент не успевает совершить оборот вокруг z-оси), а при $\omega_{p r} \tau_{e f f} \gg 1$ реализуется режим высокой добротности прецессии (магнитный момент многократно прецессирует за время эффективной спиновой релаксации).

В первом режиме при $\omega_{p r} \tau_{e f f} \ll 1$ вероятность квантовых переходов, согласно (30), равна

$$
W \approx n_{e l}\left|\frac{P_{z}(0)-P_{z}^{e}}{\tau_{\|}}\right| .
$$

Таким образом, в режиме низкой добротности прецессии вероятность переходов задается временем продольной релаксации спиновой системы свободных электронов металла, определяемым, в свою очередь, свойствами термостата. Для оценки вероятности переходов (31) примем $n_{e l}$ по порядку величины равным концентрации свободных электронов в металле $n \approx 10^{22} \mathrm{~cm}^{-3}$. Это соответствует случаю максимально возможного неравновесного состояния по спину и осуществимо при плотности тока инжекции, много большей тока диффузии [30]. В результате получаем следующую оценку вероятности переходов в единицу времени и в единице объема при $P_{z}(0)=0.4$ (железо, [4]), $P_{z}^{e}=0.2$ (пермаллой, [4]), $\tau_{\|}=10^{-13} \mathrm{~s}$

$$
W \approx 10^{34} \mathrm{~s}^{-1} \mathrm{~cm}^{-3} \text {. }
$$

Для режима высокой добротности прецессии при учтем, что приближенная формула (30) совпадает с численным решением системы $(27)$ при $t \approx 10 \tau_{\|}$. В этом случае вероятность переходов, как следует из (33), равна

$$
W \approx n_{e l}\left|10\left(P_{z}(0)\right) \gamma^{2} N_{A}^{2(\perp)} \tau_{\perp}\right| \approx 10^{37} \mathrm{~s}^{-1} \mathrm{~cm}^{-3}
$$

и определяется поперечным временем спиновой релаксации. Для оценки (32) принималось, что $n_{e} \approx 10^{22} \mathrm{~cm}^{-3}$ (случай максимально возможного неравновесного состояния по спину [30]), $\omega_{p r} \approx 6 \cdot 10^{14} \mathrm{~s}^{-1}, \tau_{\|} \approx 10^{-13} \mathrm{~s}[6,7]$ и $\tau_{\perp} \approx 10^{-15} \mathrm{~s}[15]$.

Из формулы (29) следуют интересные свойства процессов релаксации. При $\omega_{p r} \tau_{e f f} \gg 1$ и $t \gg \tau_{\|} / \omega_{p r} \tau_{e f f}$ заселенности верхнего и нижнего электронных подуровней выравниваются. В режиме высокой добротности прецессии, т.е. при достаточно большом внешнем электромагнитном поле $\left(H_{A}^{(\perp)} / H_{s d}^{(z)}>0.1\right)$, скорость поступления энергии от поля может превосходить скорость отбора энергии колебаний магнитного момента термостатом. В результате компенсируется отбор и отдача энергии электромагнитному полю осциллятором, система переходит в состояние равновесия с действующим полем и имеет место так называемый эффект насыщения [35].

Совпадение численного расчета [19] с приближенным значением $W$ из формулы (32) получается лишь при 
$H_{A}^{(\perp)}=0$. Тем не менее, все приведенные выше выводы, следующие из приближенных формул (30)-(32), подтверждаются точным численным расчетом. Чем выше значение $\omega_{p r} \tau_{e f f}$, тем больше вероятность переходов при $t=0$. При достаточно большом значении $\omega_{p r} \tau_{e f f}$ заселенности верхнего и нижнего электронных подуровней выравниваются с течением времени и вероятность переходов $W$ стремится к нулю.

\section{6. Оценка степени анизотропии среды излучения}

Для выяснения соотношения вкладов компонент $\mathbf{H}_{A}$ и $\mathrm{n} \mathbf{H}_{H}$ из (24), определяемых электромагнитной волной, в интенсивность излучательных переходов с переворотом спина, рассмотрим теперь противоположный случай, когда среда излучения анизотропна в малой степени. Для этого оценим отношение перпендикулярных к встроенному эффективному $s d$-обменному полю $\mathbf{H}_{s d}^{0}$ компонент $\left|\mathbf{H}_{A}^{\perp}\right| /\left|\mathbf{H}_{H}^{\perp}\right|$. Учтем, что $\mathbf{H}_{H}=\operatorname{rot} \mathbf{A}=\mathbf{k}_{x} \mathbf{A}$ для высокочастотной плоской волны с волновым вектором $\mathbf{k}$, при магнитной проницаемости средч, равной единице, и при калибровке векторного потенциала, определенной как $\operatorname{div} \mathbf{A}=(\mathbf{k}, \mathbf{A})=0$. Для упрощения рассмотрим случай, когда электромагнитная волна распространяется вдоль эффективного $s d$-обменного поля $\mathbf{H}_{s d}^{0}$. В этом случае как вектор $\mathbf{H}_{H}$, так и векторный потенциал $\mathbf{A}$, перпендикулярны ему. Поэтому

$$
\left|\mathbf{H}_{H}^{\perp}\right|=\left|\mathbf{H}_{H}\right|=|\mathbf{k} \| \mathbf{A}| .
$$

Соответственно величина перпендикулярной компоненты $\mathbf{H}_{A}^{\perp}$ определяется с учетом (5) и (7), а также зависит от анизотропии среды излучения. Для ее определения найдем проекции векторов $\mathbf{H}_{s d}^{0}$ и $\mathbf{H}_{A}$ на главные оси тензора $s d$-обмена. В этих осях

$$
\left(\mathbf{H}_{s d}^{0}\right)_{i}=\left(\alpha_{s d}\right)_{i i}\left(\mathbf{M}_{2}\right)_{i}
$$

и

$$
\left(\mathbf{H}_{A}\right)_{i}=\frac{e}{2 c}\left(M_{2}\right)_{i} \sum_{k}\left(\frac{\partial\left(\alpha_{s d}\right)_{i i}}{\partial p_{k}} A_{k}+A_{k} \frac{\partial\left(\alpha_{s d}\right)_{i i}}{\partial p_{k}}\right) .
$$

В отсутствие данных, позволяющих на микроскопическом уровне определить тензор $s d$-обмена, используем следующую оценку производной компонент тензора $s d$-обмена:

$$
\frac{\partial\left(\alpha_{s d}\right)_{i i}}{\partial p_{k}}=\gamma_{i}\left(\alpha_{s d}\right)_{i i} \frac{a_{k}}{\hbar},
$$

где $\gamma_{i}$ - компоненты единичного вектора, учитывающего анизотропию производной компонент тензора $s d$-обмена, а $a_{k}$ - периоды решетки вдоль его главных осей. Подстановка (36) в (35) с учетом (34) дает

$$
\left(\mathbf{H}_{A}\right)_{i}=\frac{e}{c \hbar}\left(\mathbf{H}_{s d}^{0}\right)_{i} \gamma_{i}(\mathbf{a}, \mathbf{A})
$$

где вектор а имеет компоненты $a_{k}$ из (36). Отметим, что неколлинеарность векторов $\mathbf{H}_{s d}^{0}$ и $\mathbf{H}_{A}$ определяется вектором $\gamma$, что означает необходимость учета дополнительной анизотропии среды излучения, возникающей из производных компонент тензора $s d$-обмена по импульсу электрона в (36). Введя угол между векторами $\gamma$ и $\mathbf{H}_{s d}^{0}$, а также между векторами а и $\mathbf{A}$ как $\theta_{\gamma}$ и $\theta_{a}$, соответственно, получаем приближенно искомое отношение перпендикулярных компонент векторов $\mathbf{H}_{A}$ и $\mathbf{H}_{H}$ в рассматриваемой геометрии распространения электромагнитной волны

$$
\frac{\left|\mathbf{H}_{A}^{\perp}\right|}{\left|\mathbf{H}_{H}^{\perp}\right|}=\frac{e\left|\mathbf{H}_{s d}^{0}\right||a|}{c \hbar|k|} \sin \theta_{\gamma} \cos \theta_{a} .
$$

Для удобства оценки первого множителя в правой части формулы (38) перепишем его, введя фермиевский волновой вектор $k_{F}$ и энергию Ферми $E_{F}$ для среды инжекции. Тогда

$$
F=\frac{e\left|\mathbf{H}_{s d}^{0}\right||\mathbf{a}|}{c \hbar|\mathbf{k}|}=\frac{2 \mu_{B}\left|\mathbf{H}_{s d}^{0}\right|}{E_{F}} \frac{k_{F}^{2}|\mathbf{a}|}{2|\mathbf{k}|} .
$$

Считая, что отношение энергии $s d$-обмена к энергии Ферми порядка величины 0.01, $|\mathbf{a}| \sim 0.3 \mathrm{~nm}$, $k_{F} \sim \pi /(2|a|)$, длина волны $\lambda \sim 100 \mu \mathrm{m}$, a $|\mathbf{k}|=2 \pi / \lambda$, получаем оценку множителя $F \sim 5 \cdot 10^{2}$. Считая, что анизотропия по углу $\theta_{a}$ приводит к незначительному изменению $\cos \theta_{a}$ в (38), как следует из этой формулы с учетом оцененного множителя $F$, при угле между векторами $\mathbf{H}_{A}$ и $\mathbf{H}_{s d}^{0}$, в два раза большем чем $\theta_{a}=2 \cdot 10^{-3}$, преобладающими оказываются переходы электронов с переворотом спина под действием встроенного поля $\mathbf{H}_{A}$.

\section{7. Заключение}

В натсоящей работе получено уравнение движения магнитного момента, усредненного по ансамблю неравновесных спин-инжектированных электронов в ферромагнитном переходе, с учетом обменного взаимодействия, взаимодействия с внешним электромагнитным полем и термостатом, а также с учетом пространственной неоднородности распределения носителей заряда. Учет пространственной неоднородности распределения носителей заряда приводит к наличию в уравнении динамики магнитного момента слагаемого, определяемого дивергенцией от плотности спинового потока, что позволяет описывать различные процессы переноса (диффузия, дрейф) электронов в магнитном переходе. Показано, что анизотропия обменного взаимодействия и наличие внешнего поля существенно влияют на вероятность энергетических переходов электронов с переворотом спина. Если в магнетике нет анизотропии обменного взаимодействия, то поперечные компоненты обменного поля (определяемые внешним электромагнитным полем) равны нулю, а релаксация продольной компоненты магнитного момента электрона проводимости полностью определяется взаимодействием спиновой системы 
электронов проводимости с термостатом. При этом анизотропия среды излучения определяется не только анизотропией тензора $s d$-обмена, но также и дополнительной анизотропией, связанной с производными этого тензора по импульсу электронов.

Авторы с прискорбием сообщают, что во время подготовки статьи скоропостижно скончался д.ф.-м.н., зав. лаб. Г.М. Михайлов, который внес существенный вклад в эту работу.

\section{Список литературы}

[1] A. Fert, J.-M. George, H. Jaffrés, R. Mattana, P. Seneor. Europhys. News 34, 6, 227 (2003).

[2] J.F. Gregg, I. Petej, E. Jouguelet, C. Dennis. J. Phys. D 35, R121 (2002).

[3] I. Zutic, J. Fabian, S. Das Sarma. Rev. Mod. Phys. 76, 323 (2004).

[4] Concepts in Spin Electronics / Ed. S. Maekawa. Oxford University Press, N.Y. (2006).

[5] А. Ферт. УФН 178, 1336 (2008) [A. Fert. Rev. Mod. Phys. 80, 1517 (2008)]; П.А. Грюнберг. УФН 178, 1349 (2008) [P.A. Grunberg. Rev. Mod. Phys. 80, 1531 (2008)].

[6] Ю.В. Гуляев, П.Е. Зильберман, А.И. Панас, Э.М. Эпштейн. УФН 179, 359 (2009).

[7] А.К. Звездин, К.А. Звездин, А.В. Хвальковский. УФН 178, 436 (2008).

[8] G. Yu, P. Upadhyaya, Y. Fan, J.G. Alzate, W. Jiang, K.L. Wong, S. Takei, S.A. Bender, L.-T. Chang, Y. Jiang, M. Lang, J. Tang, Y. Wang, Y. Tserkovnyak, P.K. Amiri, K.L. Wang. Nature Nanotechnol. 9, 548 (2014).

[9] X. Zhou, L. Ma, Z. Shi, W.J. Fan, J.G. Zheng, R.F.L. Evans, S.M. Zhou. Phys. Rev. B 92, 060402(R) (2015).

[10] Y.-C. Lau, D. Betto, K. Rode, J.M.D. Coey, P. Stamenov. Nature Nanotechnol. 11, 758 (2016).

[11] P.E. Faria Junior, G. Xu, Y.-F. Chen, G.M. Sipahi, I. Žutić. Phys. Rev. 95, 115301 (2017).

[12] J. Walowski, M. Munzenberg. J. Appl. Phys. 120, 140901 (2016)).

[13] A. Fernandez-Pacheco, R. Streubel, O. Fruchart, R. Hertel, P. Fischer, R.P. Cowburn. Nature Commun. 8, 15756 (2017).

[14] S.S. Dhillon, M.S. Vitiell, E.H. Linfield, A.G. Davies, M.C. Hoffmann, J. Booske, C. Paoloni, M. Gensch, P. Weightman, G.P. Williams, E. Castro-Camus, D.R.S. Cumming, F. Simoens, I. Escorcia-Carranza, J. Grant, S. Lucyszyn, M. Kuwata-Gonokami, K. Konishi, M. Koch, C.A. Schmuttenmaer, T.L. Cocker, R. Huber, A.G. Markelz, Z.D. Taylor, V.P. Wallace, J. Zeitler, J. Sibik, T.M. Korter, B. Ellison, S. Rea, P. Goldsmith, K.B. Cooper, R. Appleby, D. Pardo, P.G. Huggard, V. Krozer, H. Shams, M. Fice, C. Renaud, A. Seeds, A. Stöhr, M. Naftaly, N. Ridler, R. Clarke, J.E. Cunningham, M.B. Johnston. J. Phys. D 50, 363001 (2017).

[15] J.C. Slonczewski. J. Magn. Magn. Mater. 159, L1 (1996).

[16] L. Berger. Phys. Rev. B 54, 9353 (1996).

[17] Ю.В. Гуляев, П.Е. Зильберман, Э.М. Эпштейн, Р.Дж. Эллиотт. Письма в ЖЭТФ 76, 189 (2002).

[18] В. Шокли. Теория электронных полупроводников. Приложение к теории транзисторов. ИЛ, М. (1953).
[19] Е.А. Вилков, Г.М. Михайлов, С.А. Никитов, А.Р. Сафин, М.В. Логунов, V.N. Korenivskii, С.Г. Чигарев, Л.А. Фомин. ЖЭТФ 154, 6, 1108 (2018).

[20] A.M. Kadigrobov, R.I. Shekhter, I. Aronov, S.I. Kulinich, A. Pulkin, M. Jonson. Low Temp. Phys. 37, 925 (2011).

[21] Ю.В. Гуляев, П.Е. Зильберман, С.Г. Чигарев. Радиотехника и электроника 60, 441 (2015).

[22] A. Kadigrobov, Z. Ivanov, T. Claeson, R.I. Shekhter, M. Jonson. Europhys. Lett. 67, 948 (2004).

[23] A. Kadigrobov, R.I. Shekhter, M. Jonson. Low Temp. Phys. 31, 352 (2005).

[24] A.M. Kadigrobov, R.I. Shekhter, S.I. Kulinich, M. Jonson, O.P. Balkashin, V.V. Fisun, Y.G. Naidyuk, I.K. Yanson, S. Andersson, V. Korenivski. New J. Phys. 13, 023007 (2011).

[25] R.I. Shekhter, A.M. Kadigrobov, M. Jonson, E.I. Smotrova, A.I. Nosich, V. Korenivski. Opt. Lett. 36, 2381 (2011).

[26] Ю.В. Гуляев, П.Е. Зильберман, А.И. Крикунов, А.И. Панас, Э.М. Эпштейн. Письма в ЖЭТФ 85, 192 (2007).

[27] T. Pietsch, S. Egle, M. Keller, H. Fridtjof-Pernau, F. Strigl, E. Scheer. New J. Phys. 18, 093045 (2016).

[28] Ю.В. Гуляев, П.Е. Зильберман, И.В. Маликов, Г.М. Михайлов, А.И. Панас, С.Г. Чигарёв, Э.М. Эпштейн. Письма в ЖЭТФ 93, 289 (2011).

[29] V. Korenivski, A. Iovan, A. Kadigrobov, R.I. Shekhter. Europhys. Lett. 104, 27011 (2013).

[30] Е.А. Вилков, Г.М. Михайлов, С.Г. Чигарев, Ю.В. Гуляев, В.Н. Коренивский, С.А. Никитов, А.Н. Славин. Радиотехника и электроника 61, 9, 844 (2016).

[31] С.В. Вонсовский. Магнетизм. Наука, М. (1971). 1032 с.

[32] Ю.В. Гуляев, П.Е. Зильберман, Р.Дж. Эллиотт. Радиотехника и электроника 48, 1030 (2003).

[33] К. Блум. Теория матрицы плотности и ее приложения. Мир, М. (1983). 283 с.

[34] Л.Д. Ландау, Е.М. Лифшиц. Квантовая механика. Нерелятивисткая теория. Наука, М. (1974). 752 с.

[35] Ч. Сликтер. Основы теории магнитного резонанса. Мир, M. (1967). $323 \mathrm{c}$.

Редактор Е.Ю. Флегонтова 